\title{
Gender, age, religion, and tradition influenced the smoking attitudes and behaviour of Bangladeshi and Pakistani adults
}

Bush J, White M, Kai J, et al. Understanding influences on smoking in Bangladeshi and Pakistani adults: community based, qualitative study. BMJ 2003;326:962-5.

\section{Q In Bangladeshi and Pakistani adults, what influences smoking attitudes, beliefs, values, and behaviour?}

\section{DESIGN}

Qualitative study using a community participatory approach.

\section{SETTING}

Community based setting in Newcastle upon Tyne, UK.

\section{PARTICIPANTS}

141 participants (62\% men) 18-80 years of age, including smokers and non-smokers, were recruited using purposive sampling on the basis of ethnicity, gender, age, smoking status, and occupation. 87 participants ( $58 \%$ men) were Bangladeshi, and 54 (69\% men) were Pakistani.

\section{METHODS}

13 bilingual South Asian community researchers (54\% women) who had received qualitative research training used a grounded approach to data generation and analysis. 37 participants were involved in indepth semistructured interviews, and 104 participants were involved in 24 focus groups. Interviews and focus groups were based on topic guides that included smoking behaviour, views on what influences smoking, and how smoking affects health. $60 \%$ of interviews and focus groups were held in English; the remaining interviews were held in Punjabi or Urdu (for Pakistanis) or Bengali or Sylheti (for Bangladeshis). Interviews and focus groups were audiotaped, transcribed verbatim, and translated into English as necessary. Analysis used the constant comparison method, and data generation and analysis continued until no new themes emerged.

\section{MAIN FINDINGS}

4 dominant highly interrelated themes influenced smoking attitudes and behaviour: gender, age, religion, and tradition. In men, smoking was associated with a strong sense of social acceptance, social bonding, tradition, and male identity. Smoking in Bangladeshi men was more deeply socially ingrained than in Pakistani men. In contrast, smoking in Bangladeshi and Pakistani women was associated with a strong sense of cultural taboo, stigma, and nonacceptance and was thought to affect a woman's chances of marrying. Age appeared to influence the cultural acceptability of smoking. Smoking was more acceptable in older men, and to a lesser extent, in older women. Smoking in younger people was thought to For correspondence: Dr J Bush, School of Population and Health Sciences, University of Newcastle, Newcastle upon Tyne, UK. Judith.bush@ncl.ac.uk Sources of funding: Cancer Research UK and Department of Health. be disrespectful and influenced by peer pressure, and was often hidden from elders. Varied and conflicting views existed on the acceptability of smoking within the Muslim religion. However, most participants viewed smoking in a mosque to be unacceptable. Tradition, culture, and the family appeared to have important roles in forming and cultivating norms and values related to smoking.

\section{CONCLUSION}

In Bangladeshi and Pakistani adults, 4 dominant, highly interrelated themes had an important influence on smoking attitudes and behaviour: gender, age, religion, and tradition.

\section{Commentary}

fforts to effectively provide improved healthcare to minority groups require both quantitative and qualitative data. The collection of ethnic data in the UK is a relatively recent effort, and available data are sparse. ${ }^{1}$ This qualitative study by Bush et al provides important insights about smoking behaviours in Bangladeshi and Pakistani adults. The inclusion of both men and women from 18 to $>50$ years of age is particularly important because themes identified in this study help address the current lack of information on cultural influences related to health behaviours.

As always, caution must be taken in generalising to more general populations, although this is a large sample (141 participants) for a qualitative study. The authors' purposive sampling method (snowball) included efforts to ensure a range of ages. Research efforts to also "match" the demographics, particularly sex and age, of the researcher with the participants and the use of single sex and single ethnic focus groups strengthen confidence in the study findings.

Other research involving Pakistani and Bangladeshi groups supports the implications of this study for nurses interested in health behaviour interventions among minority groups. ${ }^{2}$ Nurses working with these minority groups must tailor their work to each specific group, taking into account a complex mix of cultural influences, particularly ethnicity, gender, age, and personality factors. Particular attention may be needed for Bangladeshi men given the high percentage who reported smoking $(56 \%)$ in the study by Bush et al.

George Shuster, RN, DNSc College of Nursing, University of New Mexico Albuquerque, New Mexico, USA

1 Lodge N. The identified needs of ethnic minority groups with cancer within the community: a review of the literature. Eur $J$ Cancer Care (Engl) $2001 ; 10: 234-44$

2 Khan FA, Robinson PG, Warnakulasuriya KA, et al. Predictors of tobacco and alcohol consumption and their relevance to oral cancer control amongst people from minority ethnic communities in the South Thames health region, England. J Oral Pathol Med 2000;29:214-9. 